$$
\begin{aligned}
& \text { UCRL }-21258-89 \\
& \text { DE92 } 002178
\end{aligned}
$$

\title{
Analysis of Solvent Evaporation Effects in Spin Coating of Colloidal Oxide Suspension
}

T. J. Rehg

B. G. Higgins

July 23, 1991

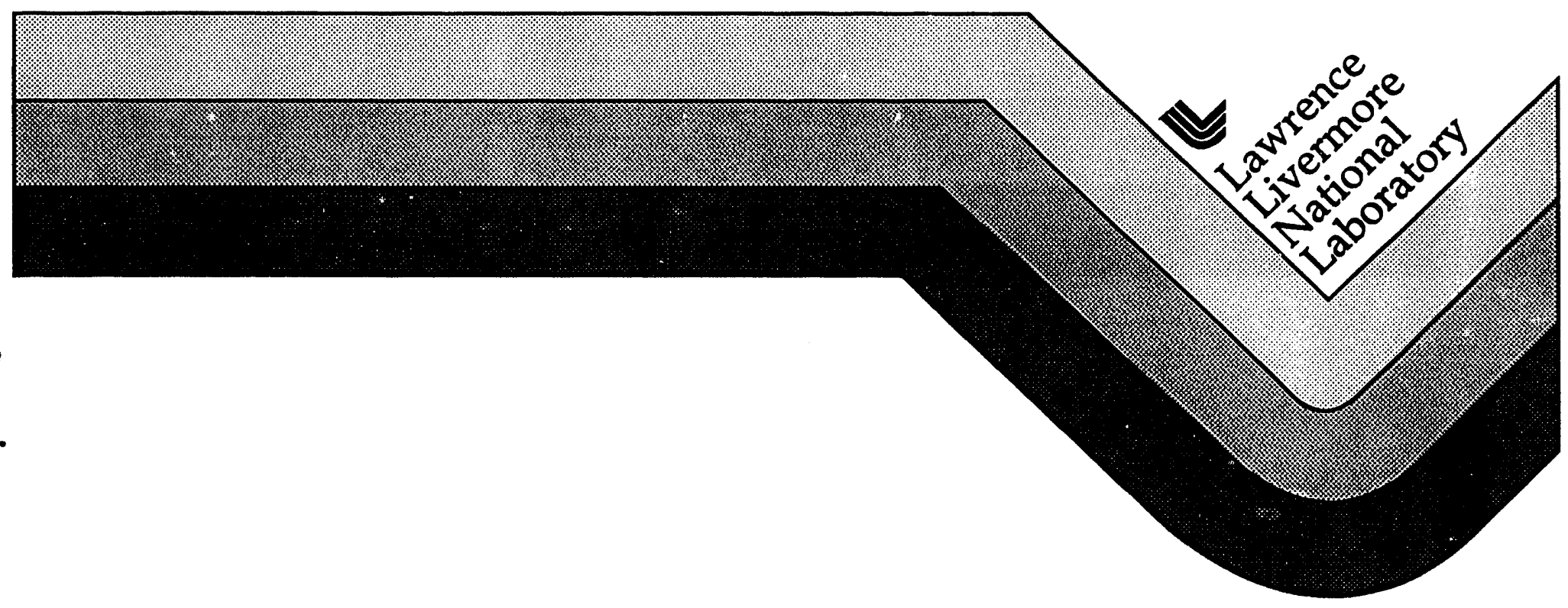

\section{MASTER}

DISTRIBUTION OF THIS DOCUMENT IS UNLIMITED 


\section{DISCLAIMER}

Work performed under the auspices of the U.S. Department of Energy by Lawrence Livermore National Laboratory under contract number W-7405-ENG-48.

This document was prepared as an account of work sponsored by an agency of the United States Government. Neither the United States Government nor the University of California nor any of their employees. makes any warranty, express or implied, or assumes any legal liability or responsibility for the accuracy, completeness, or usefulness of any information, apparatus, product, or process disclosed, or represents that its use would not infringe privately owned rights. Reference herein to any specific commercial products, process, or service by trade name, trademark, manufacturer, or otherwise, does not necessarily constitute or imply its endorsement, recommendation, or favoring by the Linited States Government or the University of California. The views and opinions of authors expressed herein do not necessarily state or reflect those of the United States Government or the University of California, and shall not be used for advertising or product endorsement purposes. 


\section{Progress Report 1989-90}

\section{Analysis of Solvent Evaporation Effects in Spin Coating of Colloidal Oxide Suspensions}

The goal of our research this past year was to develop a theory for spin coating that accounts for solvent evaporation, film hydrodynamics, and the rheology of the colloidal suspension being coated. Our efforts in this regard have been quite successful. We have developed a model of the spin coating of hard sphere colloidal suspensions. A hard sphere colloidal suspension is one where the net interaction (other than hydrodynamic) of the spheres is governed by the hard sphere potential. This model, given the necessary constitutive relations, (i.e. diffusivity and viscosity dependence on concentration) can be readily modified to include the spin coating of more complicated colloidal suspensions where both van der Waals and electrostatic forces are important (such as in the sol-gel colloidal oxide suspensions used at LLNL). This model offers, for the first time, a predictive capability for the spin coating of low viscosity solutions. The model is currently capable of predicting the film thickness and, given the constitutive relations for non-hard sphere suspensions, can be modified to predict the porosity as well.

In order to account for film flow, solvent evaporation and diffusion, and evaporative cooling it was necessary to include the time dependent momentum, continuity, convective diffusion, and thermal energy equations in the model. The physical properties are, in general, functions of temperature and concentration. As a consequence, the equation set outlined above is both non-linear and coupled. The constitutive relation for the diffusivity of the hard spheres in the suspension has been derived from first principles by Batchelor (1976) and can be shown to be equal to the diffusivity of the solvent in the suspension (given a suspension of constant density). The constitutive relation for the viscosity has been well documented in the literature and is reviewed by Russel (1989). The 
model was solved using an implicit finite difference scheme on the Cray Y/MP at the San Diego Supercomputer Center.

The model puts to rest the confusing result that some investigators observe a film thickness dependence on the inverse square root of the rotation rate and some do not. Our model shows that this dependence is a function of the acceleration rate of the spin coater. For instantaneous accelerations (and, obviously, very fast accelerations), the dependence is inverse square root. However, if the substrate is accelerated more slowly, the dependence is more complicated. The accompanying plot demonstrates this result.

The model is a useful tool for interpretating our experimental investigation into the instabilities of spin coating. We have demonstrated that the radial striations observed in spin coating are a consequence of evaporative convection (Rehg and Higgins, 1991). Evaporative convection is the convective flow which spontaneously develops in otherwise stagnant pools of volatile liquids and is driven by surface tension gradient and/or buoyancy forces induced by concentration and/or temperature gradients. It can be characterized in part by the Marangoni and Rayleigh numbers These dimensionless groups are a measure of the ratio of surface tension gradient forces to viscous forces and buoyancy forces to viscous forces, respectively. Reduction of these quantities below a critical value will result in the elimination of the radial striations. Our model of spin coating has allowed us to evaluate various spin coating protocols to determine their effect on the Marangoni and Rayleigh numbers. The results of the analysis suggest that rapid substrate acceleration rates, partial saturation of the overlying gas phase with solvent, and elevating the temperature of the overlying gas phase above that of the coating fluid will all reduce the values of the Marangoni and Rayleigh numbers. Our experiments also suggest that the presence of surfactant yield striation free coatings.

In order to fully understand the conditions necessary to eliminate striations for an arbitrary spin coating system, a linear stability analysis is required. The model described 
above provides the necessary base flow for this analysis. The linear stability analysis is described in more detail in the accompanying proposal.

As a consequence of our work this past year, we will publish two papers. The first, entitled Evaporative Convection in Spin Coating. concerns our experiments on the stability of spin coating and was outlined in our presentation at the 1990 Spring AIChE meeting in Orlando. It is to be submitted to Physics of Fluids A. The second describes the detailed results of the model discussed here. We are presently conducting some experiments on hard sphere suspensions to accompany the model. At this time it is our intention to submit this manuscript to the AIChE Journal.

\section{References}

G. K. Batchelor, JFM, 74, 1(1976).

W. B. Russel, Colloidal Dispersions, Cambridge University Press, Cambridge, 1989.

T. J. Rehg and B. G. Higgins, Phys. Fluids $A$, to be submitted. 

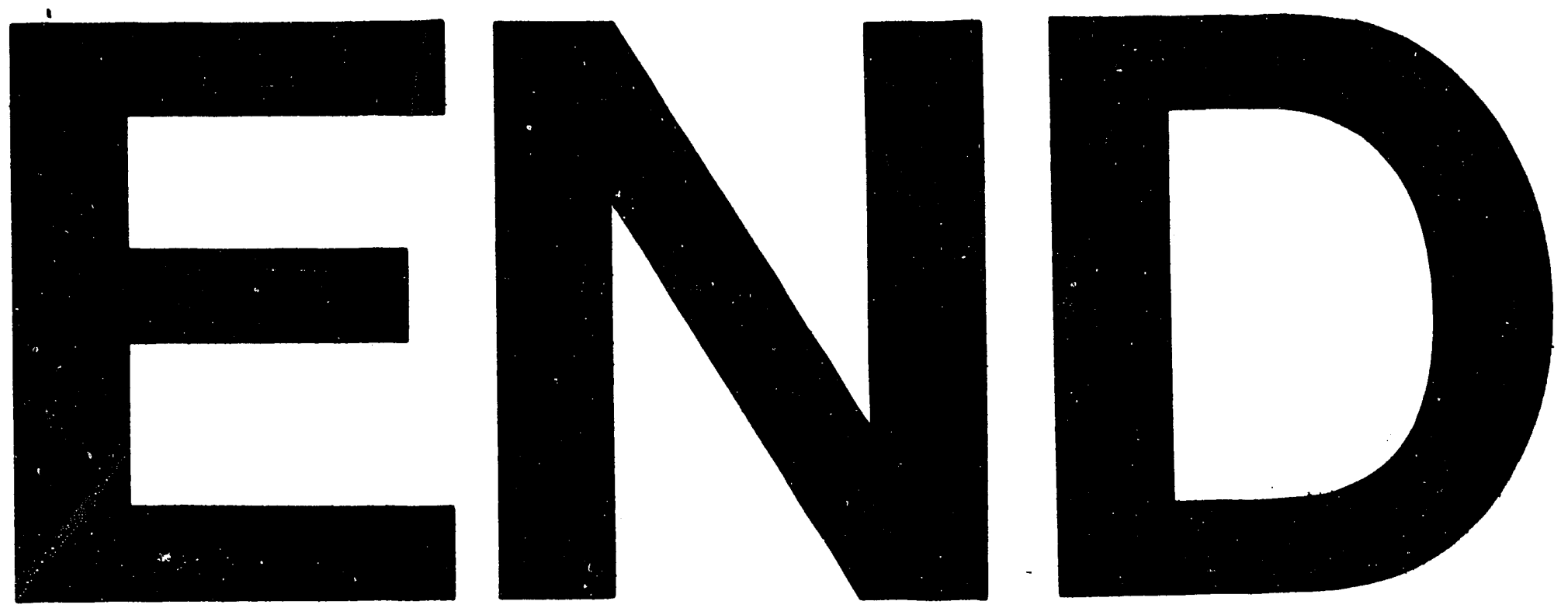

4

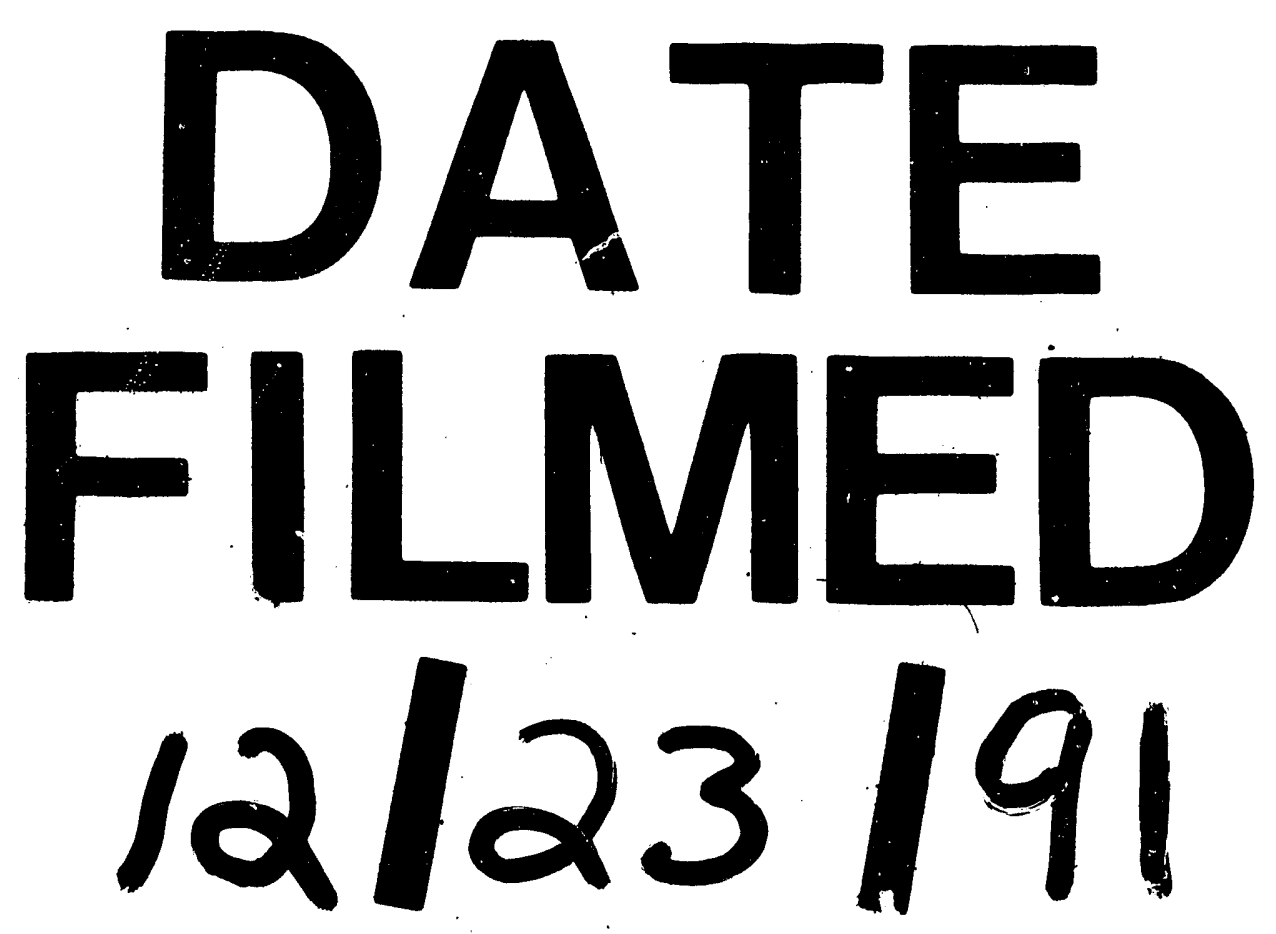

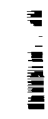

$I$ 


\section{]}

